\title{
Stability of the Feasible Set Mapping of Linear Systems with an Exact Constraint Set
}

\author{
Jorge Amaya* \\ CMM-DIM, Universidad de Chile \\ Av. Blanco Encalada 2120, Santiago, Chile \\ jamaya@dim.uchile.cl \\ Paul Bosch \\ Facultad de Ingeniería, Universidad Diego Portales \\ Av. Ejército 441, Santiago, Chile \\ paul.bosch@udp.cl \\ Miguel A. Goberna ${ }^{\dagger}$ \\ Departamento de Estadística e Investigación Operativa \\ Universidad de Alicante, 03080, Alicante, Spain \\ mgoberna@ua.es
}

\begin{abstract}
This paper deals with the stability of the feasible set mapping of linear systems of an arbitrary number (possibly infinite) of equations and inequalities such that the variable $x$ ranges on a certain fixed constraint set $X \subset \mathbb{R}^{n}$ ( $X$ could represent the solution set of a given constraint system, e.g., the positive cone of $\mathbb{R}^{n}$ in the case of sign constraints). More in detail, the paper provides necessary as well as sufficient conditions for the lower and upper semicontinuity (in Berge sense), and the closedness, of the set-valued mapping which associates, with any admissible perturbation of the given (nominal) system its feasible set. The parameter space is formed by all the systems having the same structure (i.e., the same number of variables, equations and inequalities) as the nominal one, and the perturbations are measured by means of the pseudometric of the uniform convergence.

Mathematics Subject Classification (2000): Primary: 49K40, 90C34; Secondary: 15A39, 90C05.

Key words: Stability, feasible set mapping, linear programming, linear semi-infinite programming.
\end{abstract}

*Partially supported by Fondecyt Grant 1020(7020)-646 and DI-U. de Chile Grant ENL 06/11.

${ }^{\dagger}$ Research supported by MEC and FEDER, Grant MTM2005-08572-C03-01. 


\section{Introduction}

In this paper we consider given a non-empty set $X \subset \mathbb{R}^{n}$ and a linear system (called nominal),

$$
\sigma=\left\{a_{t}^{\prime} x \geq b_{t}, t \in W ; a_{t}^{\prime} x=b_{t}, t \in E\right\}
$$

where $W$ and $E$ are arbitrary index sets (possibly empty or infinite) such that $W \cap E=\emptyset$, $T:=W \cup E \neq \emptyset, a: T \rightarrow \mathbb{R}^{n}$, and $b: T \rightarrow \mathbb{R}$ (called LHS and RHS functions, respectively). $X$ could represent either the solution set of those constraints that cannot be perturbed (e.g., sign constraints), in continuous optimization, or a discrete set (i.e., a set with no accumulation point), in combinatorial optimization.

The solution set of $\sigma$ in $X$ is

$$
F^{X}=\left\{x \in X \mid a_{t}^{\prime} x \geq b_{t}, t \in W ; a_{t}^{\prime} x=b_{t}, t \in E\right\} .
$$

In particular, the solution set of $\sigma$ in $\mathbb{R}^{n}$ is denoted with $F$, i.e., $F=F^{\mathbb{R}^{n}}$. We say that $\sigma$ is consistent (relative to $X$ ) if $F^{X} \neq \emptyset$.

This paper analyzes the effect on $F^{X}$ of small changes in the coefficients of $\sigma$ due to either computing or measurement errors, maintaining the space of variables, $\mathbb{R}^{n}$, and the sets indexing inequalities and equations, $W$ and $E$. Thus the parameter space will be the real vector space

$$
\Theta=\left\{\left(\begin{array}{l}
c \\
d
\end{array}\right) \mid c: T \rightarrow \mathbb{R}^{n}, d: T \rightarrow \mathbb{R}\right\}
$$

where we identify $\left(\begin{array}{l}c \\ d\end{array}\right) \in \Theta$ with the system

$$
\sigma_{1}=\left\{c_{t}^{\prime} x \geq d_{t}, t \in W ; c_{t}^{\prime} x=d_{t}, t \in E\right\}
$$

and consequently we will write $\sigma_{1} \in \Theta$ (observe that $\Theta$ only depends on $T$ and $n$ ). If $\sigma_{1}$ is the resulting system of perturbing $\sigma$, the size of this perturbation is measured by means of the uniform pseudometric, i.e.,

$$
d\left(\sigma_{1}, \sigma\right)=\sup _{t \in T}\left\|\left(\begin{array}{c}
c_{t} \\
d_{t}
\end{array}\right)-\left(\begin{array}{c}
a_{t} \\
b_{t}
\end{array}\right)\right\|_{\infty}
$$

Obviously, $\Theta$ is locally metrizable. Now we introduce three subsets of $\Theta$ which play a crucial role in this paper. We denote by $\Theta_{c}^{X}, \widehat{\Theta}$ and $\bar{\Theta}$ the sets of consistent systems (relative to $X$ ), the set of systems $\sigma_{1} \in \Theta$ such that the LHS function $c: T \rightarrow \mathbb{R}^{n}$ is bounded, and the set of systems $\sigma_{1}$ such that the coefficient function $(c, d): T \rightarrow \mathbb{R}^{n+1}$ is bounded, respectively. Obviously, $\bar{\Theta} \subset \widehat{\Theta}$ and both sets are open and closed in $\Theta$. The interiority of $\sigma$ in $\Theta_{c}^{X}$ is a kind of stability that has been analyzed in [1].

Since all the results in this paper concern the behavior of the feasible set in the proximity of the nominal system, they remain valid replacing $\Theta$ with an arbitrary neighborhood of $\sigma$, e.g., if $\left.\sigma \in \bar{\Theta},\left\{\sigma_{1} \in \Theta \mid d\left(\sigma_{1}, \sigma\right)<+\infty\right\}=\bar{\Theta}\right)$. They are also valid for a different norm in 
$\mathbb{R}^{n}$ and under re-scaling of the linear constraint of index $t \in T$ with an arbitrary weight $\beta_{t}$, provided that there exist two positive scalars $\beta$ and $\bar{\beta}$ such that $\beta \leq \beta_{t} \leq \bar{\beta}$ for all $t \in T$.

There exists a wide literature on the continuity properties of the feasible set mapping $\mathcal{F}: \Theta \rightrightarrows \mathbb{R}^{n}$ such that $\mathcal{F}\left(\sigma_{1}\right)=F_{1}$ is the solution set of $\sigma_{1}$ (relative to $\mathbb{R}^{n}$ ) in the case that $E=\emptyset$ (see, e.g., [11], [5], [10] and references therein). The recent paper [6] extends to linear systems such that $0<|E| \leq n$ results on the interiority of $\sigma$ in $\Theta_{c}^{\mathbb{R}^{n}}$ and provides formulae for the distance from $\sigma$ to ill-posedness. Many other alternative approaches to the stability of the feasible set mapping are possible, e.g., the study of the topological behavior of the feasible set in the proximity of the nominal system (see, e.g., [15] and [16]), regularity properties and error bounds (see, e.g., [17] and [18]), etc.

This paper analyzes the stability of $\sigma$, with an arbitrary index set $E$ and $\emptyset \neq X \subset \mathbb{R}^{n}$ (the same context of [1]), from the perspective of the continuity properties, at $\sigma$, of the set-valued mapping, $\mathcal{F}^{X}: \Theta \rightrightarrows \mathbb{R}^{n}$, which associates to each $\sigma_{1} \in \Theta$ the set of solutions of $\sigma_{1}$ in $X$, denoted as $\mathcal{F}^{X}\left(\sigma_{1}\right)=F_{1}^{X}=F_{1} \cap X$. In other words, $\mathcal{F}^{X}$ is the intersection mapping of $\mathcal{F}$ with the constant set-valued mapping $X$. For the sake of simplicity, we eliminate $X$ when it is the whole space $\mathbb{R}^{n}$ (e.g., $\Theta_{c}^{\mathbb{R}^{n}}, F^{\mathbb{R}^{n}}$ and $\mathcal{F}^{\mathbb{R}^{n}}$ are denoted by $\Theta_{c}, F$ and $\mathcal{F}$, respectively).

The paper is organized as follows. Section 2 introduces the necessary notation and elements of set-valued analysis, including known results on the stability of $\mathcal{F}$ when $E=\emptyset$, whereas Section 3 analyzes in an abstract framework the transference of stability properties from arbitrary multifunctions to their corresponding intersection mappings. Sections 4-6 provide necessary conditions and sufficient conditions for three desirable continuity properties of $\mathcal{F}^{X}$ (closedness, lower and upper semicontinuity). Finally, Section 7 contains the conclusions, including applications of the results in Sections 4-6 to three important linear programming (LP) models: ordinary LP problems formulated in the general form,

$$
P_{1}: \text { Min } c^{\prime} x \text { s.t. } A x \geq b, B x=d, x \in \mathbb{R}^{n},
$$

LP problems in standard format,

$$
P_{2}: \text { Min } c^{\prime} x \text { s.t. } A x=b, \quad x \in \mathbb{R}_{+}^{n},
$$

and 0-1 LP problems

$$
P_{3}: \text { Min } c^{\prime} x \text { s.t. } A x \geq b, \quad x \in\{0,1\}^{n},
$$

where $A(m \times n), b \in \mathbb{R}^{m}, B(p \times n), d \in \mathbb{R}^{p}$, and $c \in \mathbb{R}^{n}$. Observe that $X$ is closed in these models, it is convex in $P_{1}$ and $P_{2}$, and it is discrete in $P_{3}$.

\section{Preliminaries}

Let us introduce the necessary notation. Given a non-empty subset $Y$ of a certain real linear space, we denote by conv $Y$ the convex hull of $Y$. If $Y$ is convex, $\operatorname{dim} Y$ and $0^{+} Y$ represent the dimension and the recession cone, respectively. When the linear space is equipped with 
a certain topology, we denote by $\mathrm{cl} Y$, int $Y$, and rint $Y$ the closure, the interior, and the relative interior of $Y$, respectively. Instead of $\lim _{r \rightarrow \infty} y_{r}=y_{0}$ we write $y_{r} \rightarrow y_{0}$. We denote by $B(x ; \varepsilon)$ the open ball centered at $x$ with radius $\varepsilon>0$ in the Euclidean space. The zero vector in $\mathbb{R}^{n}$ is $0_{n}, I_{n}$ is the identity matrix and ker $A$ is the kernel of a linear mapping $A$.

We deal with the stability of $\mathcal{F}^{X}$ in three different senses that we define for arbitrary set-valued mappings (other related concepts can be found, e.g., in [3], [2] and [19]). Let $Y$ be an arbitrary set (called space of parameters) equipped with some locally metrizable topology, let $\mathcal{A}: Y \rightrightarrows \mathbb{R}^{n}$ and let $y_{0} \in Y$.

$\mathcal{A}$ is closed at $y_{0}$ if for any $\bar{x} \in \mathbb{R}^{n}$ and any two sequences, $\left\{y_{r}\right\} \subset Y$ and $\left\{x^{r}\right\} \subset \mathbb{R}^{n}$ such that $y_{r} \rightarrow y_{0}, x^{r} \in \mathcal{A}\left(y_{r}\right), r=1,2, \ldots$, and $x_{r} \rightarrow x_{0}$, one gets $x_{0} \in \mathcal{A}\left(y_{0}\right)$.

$\mathcal{A}$ is lower semicontinuous in Berge-Kuratowski sense (lsc in brief) at $y_{0}$ if for each open set $U$ such that $U \cap \mathcal{A}\left(y_{0}\right) \neq \emptyset$ there exists an open set $V, y_{0} \in V \subset Y$, such that $U \cap \mathcal{A}(y) \neq \emptyset$ for every $y \in V$.

$\mathcal{A}$ is upper semicontinuous in Berge-Kuratowski sense (usc) at $y_{0}$ if for each open set $U$ such that $\mathcal{A}\left(y_{0}\right) \subset U$ there exists an open set $V, y_{0} \in V \subset Y$, such that $\mathcal{A}(y) \subset U$ for every $y \in V$.

The mapping $\mathcal{A}$ is closed (lsc, usc) if it is closed (lsc, usc) at $y$ for all $y \in Y$.

The statement of the following well-known result (see, e.g., [3, Lemmas 2.2.1 and 2.2.3, part (i)]) involves a concept of a different kind: $\mathcal{A}$ is locally bounded at $y_{0}$ if there exists an open set $V, y_{0} \in V \subset Y$, such that the set $\bigcup_{y \in V} \mathcal{A}(y)$ is bounded.

Lemma 1 Given $\mathcal{A}: Y \rightrightarrows \mathbb{R}^{n}$ and $y_{0} \in Y$, the following statements hold:

(i) If $\mathcal{A}$ is usc at $y_{0}$ and $\mathcal{A}\left(y_{0}\right)$ is closed, then $\mathcal{A}$ is closed at $y_{0}$.

(ii) If $\mathcal{A}$ is closed and locally bounded at $y_{0}$, then $\mathcal{A}$ is usc at $y_{0}$.

The next result is Lemma 2(iii) in [13].

Lemma 2 If $\mathcal{A}$ is usc at $y_{0}$, then there exist a positive scalar $\bar{\rho}$ and a neighborhood of $y_{0}, V$, such that

$$
\mathcal{A}(y) \backslash \operatorname{cl} B\left(0_{n} ; \bar{\rho}\right) \subset \mathcal{A}\left(y_{0}\right) \backslash \operatorname{cl} B\left(0_{n} ; \bar{\rho}\right), \text { for all } y \in V \text {. }
$$

The converse statement holds when $\mathcal{A}$ is closed at $y_{0}$.

If $\mathcal{A}$ is a closed mapping (as it is $\mathcal{F}^{X}$ under mild conditions), then the images are closed and so the lsc property and the closedness of $\mathcal{A}$ at $y_{0}$ can be expressed in terms of PainlevéKuratowski limits. The corresponding properties, called inner and outer semicontinuity, are a suitable pair of stability properties (see the discussion in [19]), whereas the usc property is too restrictive, according to (1).

The lsc property of $\mathcal{F}^{X}$ is related with the following desirable properties of $\sigma \in \Theta_{c}^{X}: \sigma$ is stably consistent if $\sigma \in \operatorname{int} \Theta_{c}^{X}$ and it is RHS-stably consistent if there exists $\varepsilon>0$ such that $\left\{a_{t}^{\prime} x \geq d_{t}, t \in T\right\} \in \Theta_{c}^{X}$ for all function $d: T \rightarrow \mathbb{R}$ such that $\left|d_{t}-b_{t}\right|<\varepsilon$ for all $t \in T$. 
Obviously, if $\mathcal{F}^{X}$ is lsc at $\sigma \in \Theta_{c}^{X}$, then $\sigma$ is stably consistent, and this implies that $\sigma$ is RHS-stably consistent. The stability of $\sigma$ is also related with the existence of Strong Slater (SS in brief) points of $\sigma$ in $X$ (or at least in cl $X$ ), i.e., points $\bar{x}$ such that $a_{t}^{\prime} \bar{x} \geq b_{t}+\varepsilon$ for some $\varepsilon>0$ and for all $t \in W$, and such that $a_{t}^{\prime} \bar{x}=b_{t}$ for all $t \in E$ (if $E \neq \emptyset$ ).

Observe that, if $\bar{x}$ is SS point of $\sigma$ and $E=\emptyset$, then $\bar{x}$ is also SS point for systems close enough to $\sigma$. In fact, if $\sigma_{1}=\left\{c_{t}^{\prime} x \geq d_{t}, t \in W\right\} \in \Theta$ satisfies

$$
d\left(\sigma_{1}, \sigma\right)<\delta<\varepsilon(n+1)^{-\frac{1}{2}}\left\|\left(\begin{array}{c}
\bar{x} \\
-1
\end{array}\right)\right\|^{-1}
$$

and $t \in W$, we have

$$
\begin{aligned}
\left|\left[\left(\begin{array}{c}
c_{t} \\
d_{t}
\end{array}\right)-\left(\begin{array}{l}
a_{t} \\
b_{t}
\end{array}\right)\right]^{\prime}\left(\begin{array}{c}
\bar{x} \\
-1
\end{array}\right)\right| & \leq\left\|\left(\begin{array}{c}
c_{t} \\
d_{t}
\end{array}\right)-\left(\begin{array}{c}
a_{t} \\
b_{t}
\end{array}\right)\right\|\left\|\left(\begin{array}{c}
\bar{x} \\
-1
\end{array}\right)\right\| \\
& <\delta \sqrt{n+1}\left\|\left(\begin{array}{c}
\bar{x} \\
-1
\end{array}\right)\right\|,
\end{aligned}
$$

so that

$$
\begin{aligned}
c_{t}^{\prime} \bar{x}-d_{t} & \geq\left(a_{t}^{\prime} \bar{x}-b_{t}\right)-\delta \sqrt{n+1}\left\|\left(\begin{array}{c}
\bar{x} \\
-1
\end{array}\right)\right\| \\
& \geq \varepsilon-\delta \sqrt{n+1}\left\|\left(\begin{array}{c}
\bar{x} \\
-1
\end{array}\right)\right\| \\
& >0 .
\end{aligned}
$$

Moreover, if $\bar{x}$ is SS point of $\sigma$ and $x \in F$, then every point of the segment $] \bar{x}, x[$ is also SS point of $\sigma$. In fact, let $\varepsilon$ be as above and take $\lambda \in] 0,1[$. Denoting $z(\lambda):=(1-\lambda) x+\lambda \bar{x}$, we have

$$
a_{t}^{\prime} z(\lambda)-b_{t}=(1-\lambda) a_{t}^{\prime} x+\lambda a_{t}^{\prime} \bar{x}-b_{t} \geq \lambda \varepsilon, \text { for all } t \in W
$$

and

$$
a_{t}^{\prime} z(\lambda)-b_{t}=(1-\lambda) a_{t}^{\prime} x+\lambda a_{t}^{\prime} \bar{x}-b_{t}=0, \text { for all } t \in E,
$$

so that $z(\lambda)$ is SS point of $\sigma$. Consequently, the set of SS points of $\sigma$ is convex and dense in $F$. Next we show that, if there exists a SS point of $\sigma$ and $\sigma \in \widehat{\Theta}$, then the set of SS point of $\sigma$ is open (relatively open) if $E=\emptyset(E \neq \emptyset$, respectively). In the worst case, assume that $E \neq \emptyset$ and let

$$
L:=\left\{x \in \mathbb{R}^{n} \mid a_{t}^{\prime} x=b_{t}, t \in E\right\} .
$$

$L$ is the affine hull of $F$ and so, it is also the affine hull of the set of SS points of $\sigma$. Let $\bar{x} \in \mathbb{R}^{n}$ and $\varepsilon>0$ such that $a_{t}^{\prime} \bar{x} \geq b_{t}+\varepsilon$, for all $t \in W$, and $a_{t}^{\prime} \bar{x}=b_{t}$ for all $t \in E$, and let $k>0$ such that $\left\|a_{t}\right\|<k$ for all $t \in T$. Then it is easy to verify that $B\left(\bar{x} ; \frac{\varepsilon}{2 k}\right) \cap L$ is formed by SS points of $\sigma$.

In the examples of Section 3 we make use of some well-known stability properties of $\mathcal{F}$ in the simple case that $E=\emptyset$. In fact, $\mathcal{F}$ is closed and it is usc at $\sigma \in \Theta_{c}$ if $F$ is either bounded 
or the whole space $\mathbb{R}^{n}$. The converse statement is not true unless $|T|<\infty$ (a characterization of the usc property for general systems can be found in [5], but it is very hard to be checked in practice). The next result recalls some of the well-known conditions for $\mathcal{F}$ to be lsc at $\sigma$ $([11])$.

Lemma 3 Let $\sigma \in \Theta_{c}$ be such that $E=\emptyset$. Then the following statements are equivalent to each other:

(i) $\mathcal{F}$ is lsc at $\sigma$.

(ii) $\sigma$ is stably consistent.

(iii) $\sigma$ is RHS-stably consistent.

(iv) There exists a SS point for $\sigma$.

\section{$3 \quad$ Stability of the intersection mapping}

In this section we consider given a non-empty set $X \subset \mathbb{R}^{n}$ and a set-valued mapping $\mathcal{A}$ : $Y \rightrightarrows \mathbb{R}^{n}$, where $Y$ is equipped with some locally metrizable topology. We also consider the intersection mapping $\mathcal{A}^{X}: Y \rightrightarrows \mathbb{R}^{n}$ such that $\mathcal{A}^{X}(y):=X \cap \mathcal{A}(y)$ for all $y \in Y$. It is easy to show by means of simple examples (similar to those in [4, Chapter 6], with $\mathcal{A}=\mathcal{F}$ and $T$ finite) that no continuity property is transmitted from $\mathcal{A}$ to $\mathcal{A}^{X}$ unless $X$ satisfies a certain condition. For the sake of completness we include here some conditions which are consequence of well-known results on the intersection of two set-valued mappings.

Proposition 4 Let $\mathcal{A}$ be closed at $y_{0} \in Y$. Then $\mathcal{A}^{X}$ is closed at $y_{0}$ if $\mathcal{A}\left(y_{0}\right) \cap$ cl $X \subset X$. In particular, $\mathcal{A}^{X}$ is closed (usc) at $y_{0}$ if $X$ is closed (compact, respectively). Consequently, If $\mathcal{A}$ is a closed mapping and $X$ is closed (compact, respectively), then $\mathcal{A}^{X}$ is closed (usc, respectively).

Proof: Let $\left\{y_{r}\right\} \subset Y$ and $\left\{x_{r}\right\} \subset \mathbb{R}^{n}$ such that $y_{r} \rightarrow y_{0}, x_{r} \rightarrow x_{0}$ and $x_{r} \in \mathcal{A}^{X}\left(y_{r}\right)$, $r=1,2, \ldots$ Since $x_{r} \in \mathcal{A}\left(y_{r}\right), r=1,2, \ldots$, and $\mathcal{A}$ is closed at $y_{0}, x_{0} \in \mathcal{A}\left(y_{0}\right)$. On the other hand, since $x_{r} \in X$ for all $r, x_{0} \in \mathcal{A}\left(y_{0}\right) \cap \mathrm{cl} X \subset X$. Hence $x_{0} \in \mathcal{A}^{X}\left(y_{0}\right)$. Thus $\mathcal{A}^{X}$ is closed at $y_{0}$. If $X$ is closed, $\mathcal{A}\left(y_{0}\right) \cap \operatorname{cl} X \subset X$ obviously. If $X$ is compact, then $\mathcal{A}^{X}$ is usc at $y_{0}$ by Lemma 1, part (ii).

The second statement in Proposition 4 is also consequence of [4, Theorems 5 and 7], [14, Proposition 4] and [3, Lemmas 2.2.3 and 2.2.4].

Proposition 5 If $\mathcal{A}$ is lsc at $y_{0} \in Y$, then each of the following conditions guarantees that $\mathcal{A}^{X}$ is lsc at $y_{0}$ :

(i) $X$ is open; 
(ii) $\mathcal{A}\left(y_{0}\right)$ is a convex set such that $\emptyset \neq$ int $\mathcal{A}\left(y_{0}\right) \subset X$; and

(iii) $X$ is convex and int $\mathcal{A}^{X}\left(y_{0}\right) \neq \emptyset$.

Consequently, if $\mathcal{A}$ is lsc and $X$ is open then $\mathcal{A}^{X}$ is also lsc.

Proof: We assume that $\mathcal{A}$ is lsc at $y_{0} \in Y$. Under (iii), $\mathcal{A}^{X}$ is lsc at $y_{0}$ by straightforward application of [9, Proposition 2.2].

Under either (i) or (ii), we consider an arbitrary open set $U$ such that $U \cap \mathcal{A}^{X}\left(y_{0}\right) \neq \emptyset$, i.e., $(U \cap X) \cap \mathcal{A}\left(y_{0}\right) \neq \emptyset$.

First we assume (i), i.e., that $X$ is open.

Since $U \cap X$ is open there exists a neighborhood of $y_{0}$, say $V$, such that $(U \cap X) \cap \mathcal{A}(y) \neq \emptyset$ for all $y \in V$. Then $U \cap \mathcal{A}^{X}(y) \neq \emptyset$ for all $y \in V$.

Now we assume (ii), i.e., that $\mathcal{A}\left(y_{0}\right)$ is a convex set such that $\emptyset \neq \operatorname{int} \mathcal{A}\left(y_{0}\right) \subset X$.

As a consequence of the assumptions on $\mathcal{A}\left(y_{0}\right)$ we have $\mathcal{A}\left(y_{0}\right) \subset \operatorname{clint} \mathcal{A}\left(y_{0}\right)$. Since $U \cap$ $\mathcal{A}\left(y_{0}\right) \neq \emptyset$, there exists $z \in U \cap \operatorname{int} \mathcal{A}\left(y_{0}\right)$. Let $\varepsilon>0$ such that $B(z ; \varepsilon) \subset U \cap \operatorname{int} \mathcal{A}\left(y_{0}\right)$. Since $B(z ; \varepsilon) \cap \mathcal{A}\left(y_{0}\right) \neq \emptyset$, there exists a neighborhood of $y_{0}, V$, such that $B(z ; \varepsilon) \cap \mathcal{A}(y) \neq \emptyset$ for all $y \in V$. Then, for each $y \in V$, there exists a point $x \in B(z ; \varepsilon) \cap \mathcal{A}(y)$ satisfying $x \in B(z ; \varepsilon) \subset U \cap \operatorname{int} \mathcal{A}\left(y_{0}\right) \subset U \cap X$ and $x \in \mathcal{A}(y)$, so that $x \in U \cap \mathcal{A}^{X}(y)$. Hence we have again $U \cap \mathcal{A}^{X}(y) \neq \emptyset$ for all $y \in V$.

Statements (ii) and (iii) in Proposition 5 are also consequence of [3, Lemma 2.2.5 and Corollary 2.2.5.1]. The next example shows the independence of the alternative conditions (i)-(iii) in Proposition 5 (observe that $\mathcal{A}$ is lsc at $y_{0}$ in all cases).

Example 6 Let $n=1$ and $\sigma=\{x \geq 0\}$. Let us consider three different sets $X$ :

(a) $X=]-2,-1[\cup] 1,2[$. Obviously, (i) holds whereas (ii) and (iii) fail.

(b) $X=\mathbb{R}_{+} \cup\{-1\}$. Only (ii) holds.

(c) $X=[-1,1]$. Only (iii) holds.

Proposition 7 If $\mathcal{A}$ is usc at $y_{0} \in Y$, then each of the following conditions guarantees that $\mathcal{A}^{X}$ is usc at $y_{0}$ :

(i) $X$ is closed;

(ii) $X$ is open and $\mathcal{A}\left(y_{0}\right) \subset X$; and

(iii) $\mathcal{A}^{X}$ is closed at $y_{0}$

Consequently, if $\mathcal{A}$ is usc and $X$ is closed then $\mathcal{A}^{X}$ is also usc. 
Proof: We assume that $\mathcal{A}$ is usc at $y_{0} \in Y$. Let $U$ be an open set such that $\mathcal{A}^{X}\left(y_{0}\right) \subset U$.

(i) First we assume that $X$ is closed. Since $\mathcal{A}\left(y_{0}\right) \subset W:=U \cup\left(\mathbb{R}^{n} \backslash X\right)$, there exists a neighborhood of $y_{0}, V$, such that $\mathcal{A}(y) \subset W$ for all $y \in V$. Then $\mathcal{A}^{X}(y) \subset W \cap X=U$ for all $y \in V$.

(ii) Now we assume that $\mathcal{A}\left(y_{0}\right) \subset X$, where $X$ is open. Since $\mathcal{A}\left(y_{0}\right) \subset U \cap X$, and this is open, there exists a neighborhood of $y_{0}, V$, such that $\mathcal{A}(y) \subset U \cap X$ for all $y \in V$. In that case $\mathcal{A}^{X}(y) \subset U \cap X \subset U$ for all $y \in V$.

(iii) Finally we assume that $\mathcal{A}^{X}$ is closed at $y_{0}$. If $\mathcal{A}^{X}$ is locally bounded at $y_{0}$, then Lemma 1 applies. Otherwise, according to Lemma 2, there exist a positive scalar $\bar{\rho}$ and a neighborhood of $y_{0}, V$, such that (1) holds. Intersecting with $X$ both members of (1), we get

$$
\mathcal{A}^{X}(y) \backslash \operatorname{cl} B\left(0_{n} ; \bar{\rho}\right) \subset \mathcal{A}^{X}\left(y_{0}\right) \backslash \operatorname{cl} B\left(0_{n} ; \bar{\rho}\right) \text {, for all } y \in V .
$$

We conclude that $\mathcal{A}^{X}$ is usc at $y_{0}$ applying again Lemma 2.

Concerning condition (iii) in Proposition 7, observe that, by Proposition 4 and Lemma 1(i), the closedness of $\mathcal{A}^{X}$ at $y_{0}$ holds if $\mathcal{A}\left(y_{0}\right)$ is closed and $\mathcal{A}\left(y_{0}\right) \cap \mathrm{cl} X \subset X$ (e.g., if $X$ is closed).

Let us consider now the separation of conditions (i)-(iii) in Proposition 7 under the assumption that $\mathcal{A}$ is usc at $y_{0} \in Y$. The separation of (i) and (ii) is trivial. The next example shows that (iii) does not imply (i) or (ii).

Example 8 Let $n=1$ and $\sigma=\{0 \leq x \leq 1\}$ and let $X=]-1,2] . \mathcal{F}^{X}$ is closed at $\sigma$ by Proposition 4 whereas $X$ is neither closed nor open.

Now we assume that $\mathcal{A}\left(y_{0}\right)$ is closed. Then we have seen that (i) $\Rightarrow$ (iii). On the other hand, if (ii) holds, then $\mathcal{A}^{X}$ is usc at $y_{0}$, with $\mathcal{A}^{X}\left(y_{0}\right)=\mathcal{A}\left(y_{0}\right)$ closed, so that (iii) also holds. This means that, for the feasible set mapping $\mathcal{F}$ introduced in Section 1, condition (iii) is weaker than (i) and (ii). This is not true for arbitrary set-valued mappings.

Example 9 Let $\mathcal{A}: \mathbb{R} \rightrightarrows \mathbb{R}$ such that $\mathcal{A}(0)=]-1,1[$ and $\mathcal{A}(y)=\emptyset$ otherwise. Obviously, $\mathcal{A}$ is usc at 0 . We define now $X$ in two different ways:

(a) For $X=[-1,1]$, since $\left.\mathcal{A}^{X}(0)=\right]-1,1\left[\right.$ is not closed, $\mathcal{A}^{X}$ cannot be closed at 0 . Thus (i) $\nRightarrow($ iii).

(b) For $X=\mathcal{A}(0)$ we get the same conclusion. Hence (ii) $\nRightarrow$ (iii).

\section{Closedness of $\mathcal{F}^{X}$}

It is easy to prove that $\mathcal{F}$ is closed independently of the emptiness or not of $E$.

Proposition 10 If $F \cap c l X \subset X$, then $\mathcal{F}^{X}$ is closed at $\sigma$. The converse statement holds if $\sigma \in \widehat{\Theta}$. 
Proof: The direct statement is consequence of Proposition 4, taking into account that $\mathcal{F}$ is closed.

Now we assume that $\left\{a_{t}, t \in T\right\}$ is bounded and $F \cap \operatorname{cl} X \nsubseteq X$. Let $y \in(F \cap \operatorname{cl} X) \backslash X$. Then we can write $y=\lim _{r \rightarrow \infty} x^{r}$, with $x^{r} \in X$ for all $r \in \mathbb{N}$. We associate with each $r \in \mathbb{N}$ the vector $z^{r}:=x^{r}-y$ and the system

$$
\sigma_{r}:=\left\{a_{t}^{\prime}\left(x-z^{r}\right) \geq b_{t}, t \in T\right\} .
$$

Since $d\left(\sigma_{r}, \sigma\right) \leq\left(\sup _{t \in T}\left\|a_{t}\right\|\right)\left\|z^{r}\right\|$ and $\lim _{r \rightarrow \infty} z^{r}=0_{n}, \lim _{r \rightarrow \infty} \sigma_{r}=\sigma$. Moreover, $x^{r} \in F_{r} \cap X=$ $F_{r}^{X}$ for all $r \in \mathbb{N}$, but $y \notin F^{X}$. Therefore $\mathcal{F}^{\stackrel{r \rightarrow \infty}{X}}$ cannot be closed at $\sigma$.

Observe that, if $n=1$ and $\sigma=\{x \geq 0\}$, as in Example 6 (note that $\sigma \in \widehat{\Theta}$ ), for $X=]-2,0$ ], the set valued-mapping $\mathcal{F}^{X}$ is not closed at $\sigma$ because $F \cap \operatorname{cl} X=\{0\}$ and $0 \notin X$.

The next example shows that the boundedness assumption in the converse statement of Proposition 10, $\sigma \in \widehat{\Theta}$, is not superfluous.

Example 11 Let $X=\left\{x \in \mathbb{R}^{n} \mid x_{n}<0\right\} \cup\left\{0_{n}\right\}$ and let $\sigma \in \Theta$ such that $T$ is infinite, all the elements of $F$ are SS points of $\sigma$ and there exists $\varepsilon>0$ such that $F_{1}=[-1,1]^{n-1} \times[0,1]$ if $d\left(\sigma_{1}, \sigma\right)<\varepsilon$ (according to Example 1 in [8] such a system exists due to the infiniteness of $T$ ). Since $F_{1}^{X}=\left\{0_{n}\right\}$ if $d\left(\sigma_{1}, \sigma\right)<\varepsilon, \mathcal{F}^{X}$ is constant and has closed images on a neighborhood of $\sigma$ and so it is stable in all sense at $\sigma$. Nevertheless $F \cap$ cl $X=[-1,1]^{n-1} \times\{0\} \nsubseteq X$. The reason is that necessarily $\sigma \notin \widehat{\Theta}$.

Corollary $12 \mathcal{F}^{X}$ is closed if and only if $X$ is closed.

Proof: The direct statement follows from Proposition 4. For the converse statement, consider the consistent system $\sigma_{1}:=\left\{0_{n}^{\prime} x \geq-1, t \in T\right\}$. The closedness of $\mathcal{F}^{X}$ at $\sigma_{1}$ implies the closedness of the image $F_{1}^{X}=\mathbb{R}^{n} \cap X=X$.

\section{Lower semicontinuity of $\mathcal{F}^{X}$}

We give first a sufficient condition for $\mathcal{F}^{X}$ to be lsc at $\sigma$.

Proposition 13 Let $\sigma \in \Theta$ and assume that $\sigma \in \widehat{\Theta}$ and $\left\{a_{t}, t \in E\right\}$ is linearly independent if $E \neq \emptyset$. If $X$ is a convex set such that int $X$ contains some $S S$ point of $\sigma$, then $\mathcal{F}^{X}$ is lsc at $\sigma$.

Proof: Let $U$ be an open set such that $F^{X} \cap U \neq \emptyset$. Let $\bar{x} \in F^{X} \cap U$. We discuss four possible cases.

Case 1: $E=\emptyset$.

We have $\sigma=\left\{a_{t}^{\prime} x \geq b_{t}, t \in W\right\}$ and $\widehat{x} \in \operatorname{int} X$ such that $\widehat{x}$ is SS point of $\sigma$. The segment ] $\bar{x}, \widehat{x}[$ is formed by SS points of $\sigma$ and it is contained in int $X$ by the accessibility lemma. So ] $\bar{x}, \widehat{x}[$ contains a SS point of $\sigma$, say $\widetilde{x}$, such that $\widetilde{x} \in U \cap$ int $X$. Let $\delta>0$ such that $\widetilde{x}$ is SS 
point of $\sigma_{1}=\left\{c_{t}^{\prime} x \geq d_{t}, t \in W\right\}$ if $d\left(\sigma_{1}, \sigma\right)<\delta$. In such a case $\widetilde{x} \in F_{1} \cap U \cap$ int $X \subset F_{1}^{X} \cap U$, where $F_{1}$ is the solution set of $\sigma_{1}$. Thus $F_{1}^{X} \cap U \neq \emptyset$.

Case 2: $W=\emptyset$ and $|E|=n$.

We have $\sigma=\left\{a_{t}^{\prime} x=b_{t}, t \in E\right\}$, where $\left\{a_{t}, t \in E\right\}$ is a basis of $\mathbb{R}^{n}$. By continuity of the determinant as a function of the entries, there exists $\varepsilon_{1}>0$ such that, if $\sigma_{1}=\left\{c_{t}^{\prime} x=d_{t}, t \in E\right\}$ satisfies $d\left(\sigma_{1}, \sigma\right)<\varepsilon_{1}$, then $\left\{c_{t}, t \in E\right\}$ is also a basis of $\mathbb{R}^{n}$. In such a case, there exists a unique solution of $\sigma_{1}$, say $x(c, d)$. The assumption implies that $x(a, b)=\bar{x}$ is SS point of $\sigma$ and $\bar{x} \in U \cap$ int $X$. By continuity of $x(\cdot, \cdot)$ at $(a, b)$ (recall Cramer's rule), there exists $\varepsilon_{2}$, with $0<\varepsilon_{2}<\varepsilon_{1}$, such that $x(c, d) \in U \cap$ int $X$ (and so $\left.F_{1}^{X} \cap U \neq \emptyset\right)$ if $d\left(\sigma_{1}, \sigma\right)<\varepsilon_{2}$.

Case 3: $W=\emptyset$ and $|E|<n$.

We can assume that $E=\{1, \ldots, m\}$, with $m<n$. Let $\left\{a_{m+1}, \ldots, a_{n}\right\} \subset \mathbb{R}^{n}$ such that $\left\{a_{1}, \ldots, a_{n}\right\}$ is a basis of $\mathbb{R}^{n}$. Let $b_{t}:=a_{t}^{\prime} \bar{x}, t=m+1, . ., n$. Since $\bar{x} \in X$ is solution of the system $\widetilde{\sigma}:=\left\{a_{t}^{\prime} x=b_{t}, t=1, . ., n\right\}$, with solution set $\widetilde{F}$, we have $\widetilde{F}^{X} \cap U \neq \emptyset$. Taking into account that $\widetilde{\sigma}$ is in case 2 , there exists $\varepsilon>0$ such that, if $\widetilde{\sigma}_{1}=\left\{c_{t}^{\prime} x=d_{t}, t=1, . ., n\right\}$ satisfies $d\left(\widetilde{\sigma}_{1}, \widetilde{\sigma}\right)<\varepsilon$, then $\widetilde{F}_{1}^{X} \cap U \neq \emptyset$.

Now consider an arbitrary system $\sigma_{1}=\left\{c_{t}^{\prime} x=d_{t}, t=1, . ., m\right\}$, with solution set $F_{1}$, such that $d\left(\sigma_{1}, \sigma\right)<\varepsilon$. Associating with $\sigma_{1}$ the system

$$
\widetilde{\sigma}_{1}:=\left\{c_{t}^{\prime} x=d_{t}, t=1, . ., m ; a_{t}^{\prime} x=b_{t}, t=m+1, . ., n\right\},
$$

we have $d\left(\widetilde{\sigma}_{1}, \widetilde{\sigma}\right)<\varepsilon$, so that $\widetilde{F}_{1}^{X} \cap U \neq \emptyset$. Observing that $\widetilde{F}_{1}^{X} \subset F_{1}^{X}$, we conclude that $F_{1}^{X} \cap U \neq \emptyset$.

Case 4: $W \neq \emptyset$ and $E \neq \emptyset$.

Let $k>0$ such that $\left\|a_{t}\right\|<k$ for all $t \in T$. Since there exists a SS point of $\sigma$ contained in int $X$, say $\widehat{x}$, and $] \bar{x}, \widehat{x}[\subset$ int $X$ is formed by SS points of $\sigma$, we can assume without loss of generality that $\widehat{x}$ is a SS point of $\sigma$ contained in $U \cap$ int $X$. Let $\varepsilon>0$ such that $a_{t}^{\prime} \widehat{x} \geq b_{t}+\varepsilon$ for all $t \in W$ and $a_{t}^{\prime} \widehat{x}=b_{t}$ for all $t \in E$. Let $\rho>0$ such that $\rho<\frac{\varepsilon}{2 k}$ and $B(\widehat{x} ; \rho) \subset U \cap$ int $X$.

Given $t \in W$, if $x \in B(\widehat{x} ; \rho)$, we have

$$
\begin{aligned}
a_{t}^{\prime} x-b_{t} & =a_{t}^{\prime} \widehat{x}-b_{t}+a_{t}^{\prime}(x-\widehat{x}) \\
& \geq \varepsilon-k\|x-\widehat{x}\| \\
& \geq \frac{\varepsilon}{2}
\end{aligned}
$$

Thus $B(\widehat{x} ; \rho)$ is formed by SS points of the system $\sigma^{W}:=\left\{a_{t}^{\prime} x \geq b_{t}, t \in W\right\}$. Let $\left\{x_{1}, \ldots, x_{n+1}\right\} \subset B(\widehat{x} ; \rho)$ such that $\widehat{x}$ is an interior point of conv $\left\{x_{1}, \ldots, x_{n+1}\right\}$. Let $V:=$ int conv $\left\{x_{1}, \ldots, x_{n+1}\right\}$.

Given $j \in\{1, \ldots, n+1\}$, there exists $\gamma_{j}>0$ such that $x_{j}$ is SS point of every perturbation of $\sigma^{W}, \sigma_{1}^{W}$, such that $d\left(\sigma_{1}^{W}, \sigma^{W}\right)<\gamma_{j}$. Let $\gamma:=\min \left\{\gamma_{1}, \ldots, \gamma_{n+1}\right\}>0$. Then, if $d\left(\sigma_{1}^{W}, \sigma^{W}\right)<\gamma$, $x_{1}, \ldots, x_{n+1}$ are SS points of $\sigma_{1}^{W}$, in which case $V \subset F_{1}^{W} \cap U \cap \operatorname{int} X$, where $F_{1}^{W}$ denotes the solution set of $\sigma_{1}^{W}$..

On the other hand, if $F^{E}$ denotes the solution set of $\sigma^{E}=\left\{a_{t}^{\prime} x=b_{t}, t \in E\right\}$, we have $\widehat{x} \in F^{E} \cap V$. By cases 1 and 2 , since $\left\{a_{t}, t \in E\right\}$ is linearly independent, there exists $\mu>0$ 
such that $F_{1}^{E} \cap V \neq \emptyset$ if $d\left(\sigma_{1}^{E}, \sigma^{E}\right)<\mu$, where $F_{1}^{E}$ denotes the solution set of $\sigma_{1}^{E}=\left\{c_{t}^{\prime} x=\right.$ $\left.d_{t}, t \in E\right\}$.

Consequently, if $d\left(\sigma_{1}, \sigma\right)<\min \{\gamma, \mu\}$, since

$$
d\left(\sigma_{1}, \sigma\right)=\max \left\{d\left(\sigma_{1}^{E}, \sigma^{E}\right), d\left(\sigma_{1}^{W}, \sigma^{W}\right)\right\},
$$

we get

$$
\emptyset \neq V \cap F_{1}^{E} \subset\left(U \cap \operatorname{int} X \cap F_{1}^{W}\right) \cap F_{1}^{E} \subset U \cap F^{X}
$$

This completes the proof.

The next result is the extension of Lemma 3 to systems with an arbitrary $E$ (maintaining $\left.X=\mathbb{R}^{n}\right)$.

Proposition 14 Let $\sigma \in \Theta_{c}$ be such that $\sigma \in \bar{\Theta}$ if $E \neq \emptyset$. Then the following statements are equivalent to each other:

(i) $\mathcal{F}$ is lsc at $\sigma$.

(ii) $\sigma$ is stably consistent.

(iii) $\sigma$ is RHS-stably consistent.

(iv) There exists a SS point for $\sigma$ and $\left\{a_{t}, t \in E\right\}$ is linearly independent if $E \neq \emptyset$.

Proof: We can assume $E \neq \emptyset$ (otherwise we have Lemma 3 ). (i) $\Rightarrow$ (ii) is trivial and the equivalence of (ii), (iii) and (iv) has been shown in [1, Corollary 1]. Thus it is enough to prove that (iv) $\Rightarrow(\mathrm{i})$. But this is straightforward consequence of Proposition 13 take $\left(X=\mathbb{R}^{n}\right)$.

The next two results provide sufficient conditions for $\mathcal{F}^{X}$ to lsc at a given $\sigma$ under different assumptions. The first one is immediate consequence of Propositions 5 and 14 .

Corollary 15 If there exists a SS point for $\sigma$ and, moreover, $\sigma \in \bar{\Theta}$ and $\left\{a_{t}, t \in E\right\}$ is linearly independent if $E \neq \emptyset$, then each of the following conditions guarantees that $\mathcal{F}^{X}$ is lsc at $\sigma$ :

(a) $X$ is open;

(b) $\emptyset \neq \operatorname{int} F \subset X$; and

(c) $X$ is convex and int $F^{X} \neq \emptyset$.

Proposition 16 Let $\sigma \in \Theta_{c}^{X}$ be such that $E=\emptyset$. Then each of the following conditions guarantees that $\mathcal{F}^{X}$ is lsc at $\sigma$ :

(i) $F^{X}$ contains a dense subset of SS points of $\sigma$. 
(ii) $X$ contains at least one SS point of $\sigma$ and $F^{X}$ is convex.

(iii) Every element of $F^{X}$ is SS point of $\sigma$.

Proof: First we prove that (i) implies that $\mathcal{F}^{X}$ is lsc at $\sigma$. Let $U$ be an open set in $\mathbb{R}^{n}$ such that $U \cap F^{X} \neq \emptyset$. Since the set of SS points of $\sigma$ is dense in $F, U$ contains some SS point of $\sigma$. Let $\bar{x} \in U \cap F^{X}$ and $\varepsilon>0$ such that $a_{t}^{\prime} \bar{x} \geq b_{t}+\varepsilon$ for all $t \in T$. Let $\delta>0$ such that $d\left(\sigma_{1}, \sigma\right)<\delta$ implies that $\bar{x}$ is SS point of $\sigma_{1}$. Since $\bar{x} \in U \cap F_{1}^{X}$, we get $U \cap F_{1}^{X} \neq \emptyset$. Hence $\mathcal{F}^{X}$ is lsc at $\sigma$.

Now we prove that (ii) $\Rightarrow(\mathrm{i})$. Let $\bar{x} \in X$ and $\varepsilon>0$ such that $a_{t}^{\prime} \bar{x} \geq b_{t}+\varepsilon$ for all $t \in T$, and assume that $F^{X}$ is convex. We shall prove that $Z:=\bigcup\{] \bar{x}, x\left[\mid x \in F^{X}\right\}$ is a dense subset of $F^{X}$ formed by SS points of $\sigma$. Every element of $Z$ is SS point of $\sigma$ because each segment ] $\bar{x}, x\left[\right.$, with $x \in F^{X}$, is formed by SS points of $\sigma$. On the other hand, since $\bar{x} \in F^{X}$ and this is convex, $Z \subset F^{X}$. Thus, $Z$ is a subset of $F^{X}$. Moreover, given $x \in F^{X},[\bar{x}, x] \subset$ cl $Z$, so that $F^{X} \subset$ cl $Z$, i.e., $Z$ is dense in $F^{X}$.

(iii) Let $U$ be an open set of $\mathbb{R}^{n}$ such that $U \cap F^{X} \neq \emptyset$. Select $\bar{x} \in U \cap F^{X}$. By assumption, $\bar{x}$ is SS point of $\sigma$. Take $\delta>0$ as in the proof of part (i). Since $\bar{x}$ solves $\sigma_{1}$ if $d\left(\sigma_{1}, \sigma\right)<\delta$, we get $\bar{x} \in U \cap F_{1}^{X}$.

If $E=\emptyset$ and $|T|<\infty$, the set of SS points of $\sigma$ is int $F$, so that, by the two previous results, any of the following conditions guarantees that $\mathcal{F}^{X}$ is lsc at $\sigma$ : (a) int $F \neq \emptyset$ and $X$ is open; (b) $\emptyset \neq$ int $F \subset X$; (c) int $(F \cap X) \neq \emptyset$ and $X$ is convex; (i) $F \cap X$ contains a dense subset of $F$ and int $F \neq \emptyset$; (ii) $X \cap$ int $F \neq \emptyset$ and $F \cap X$ is convex; and (iii) $F \cap X \subset$ int $F$. In this particular case it is possible to separate these conditions (recalling that (ii) $\Rightarrow(\mathrm{i})$ ).

Proposition 17 Let $\mathcal{F}^{X}$ be lsc at $\sigma \in \Theta_{c}^{X}$. Then the following statements hold:

(i) $\left\{a_{t}, t \in E\right\}$ is linearly independent if $E \neq \emptyset$;

(ii) $F$ and $X$ cannot be separated by hyperplane if $\sigma \in \widehat{\Theta}$;

(iii) cl $X$ contains some SS point of $\sigma$ if $\sigma \in \bar{\Theta},|E|<\infty$, and $X$ is convex ;

(iv) $X$ contains at least one $S S$ point of $\sigma$ if $\sigma \in \bar{\Theta},|E|<\infty$, and $X$ is a closed convex set ; and

(v) every element of $F^{X}$ is $S S$ point of $\sigma$ if $X$ is discrete.

Proof: (i)-(iv) are straightforward consequence of [1, Propositions 1 and 2] (recall that $\sigma$ is RHS-stably consistent if $\mathcal{F}^{X}$ be lsc at $\sigma \in \Theta_{c}^{X}$ ).

(v) Let $\bar{x} \in F^{X}$. Since $\bar{x}$ is an isolated point of $X$, there exists an open set in $\mathbb{R}^{n}, U$, such that $U \cap X=\{\bar{x}\}$. Obviously, $U \cap F^{X} \neq \emptyset$, so that there exists $\varepsilon>0$ such that $U \cap F_{1}^{X} \neq \emptyset$ if $d\left(\sigma_{1}, \sigma\right) \leq \varepsilon$, in which case $U \cap F_{1}^{X}=\{\bar{x}\}$. 
Assume that $\bar{x}$ is not a SS point of $\sigma$. Since $a_{t}^{\prime} \bar{x}=b_{t}$ for all $t \in E$, there exists $s \in W$ such that $a_{s}^{\prime} \bar{x}<b_{s}+\varepsilon$. Consider

$$
\sigma_{1}=\left\{c_{t}^{\prime} x \geq d_{t}, t \in W ; c_{t}^{\prime} x=d_{t}, t \in E\right\}
$$

such that $c:=a, d_{t}:=b_{t}$ for $t \in T \backslash\{s\}$, and $d_{s}:=b_{s}+\varepsilon$. Then we have $d\left(\sigma_{1}, \sigma\right)=\varepsilon$ and $\bar{x} \notin F_{1}$, in contradiction with $F_{1} \cap(U \cap X)=\{\bar{x}\}$.

Finally, we characterize the lsc property of $\mathcal{F}^{X}$ in two particular cases as a straightforward consequence of Propositions 16 and 17.

Corollary 18 Let $\sigma \in \Theta_{c}^{X}$ such that $E=\emptyset$. Then the following statements hold:

(i) If $\sigma \in \bar{\Theta}$ and $X$ is a closed convex set, then $\mathcal{F}^{X}$ is lsc at $\sigma$ if and only if $X$ contains at least one $S S$ point of $\sigma$.

(ii) If $X$ is discrete, then $\mathcal{F}^{X}$ is lsc at $\sigma$ if and only if every element of $F^{X}$ is an SS point of $\sigma$.

\section{Upper semicontinuity of $\mathcal{F}^{X}$}

The first result in this section is the usc counterpart of Proposition 14 (i.e., for the case $\left.X=\mathbb{R}^{n}\right)$.

Proposition 19 Let $\sigma \in \Theta_{c}$ such that $F \neq \mathbb{R}^{n}$. If $F$ is bounded, then $\mathcal{F}$ is usc at $\sigma$. The converse statement holds if $\sigma \in \widehat{\Theta}$.

Proof: Assume that $F$ is bounded. Let $U$ be an open set such that $F \subset U$.

Let $S:=W \cup(E \times\{1,2\})$. We associate with each function $(c, d): T \rightarrow \mathbb{R}^{n+1}$, another one $(c, d): S \rightarrow \mathbb{R}^{n+1}$ just defining

$$
\left(\begin{array}{c}
c_{(t, 1)} \\
d_{(t, 1)}
\end{array}\right)=\left(\begin{array}{c}
c_{t} \\
d_{t}
\end{array}\right) \text { and }\left(\begin{array}{c}
c_{(t, 2)} \\
d_{(t, 2)}
\end{array}\right)=-\left(\begin{array}{c}
c_{t} \\
d_{t}
\end{array}\right) \text { for all } t \in E .
$$

This way we associate with $\sigma$ another system $\widetilde{\sigma}=\left\{a_{s}^{\prime} x \geq b_{s}, s \in S\right\}$, whose solution set is $\widetilde{F}=F$. Let $(\widetilde{\Theta}, \widetilde{d})$ be the pseudometric parameter space associated with $\widetilde{\sigma}$ and let $\widetilde{\mathcal{F}}$ be the corresponding feasible set mapping. Since $\widetilde{\mathcal{F}}$ is usc at $\widetilde{\sigma}$ due to the compactness of $\widetilde{F}$, there exists $\varepsilon>0$ such that $\widetilde{F}_{1} \subset U$ whenever $\widetilde{d}\left(\widetilde{\sigma}_{1}, \widetilde{\sigma}\right)<\varepsilon$.

Thus, if $\sigma_{1} \in \Theta$ satisfies $d\left(\sigma_{1}, \sigma\right)<\varepsilon$, then $\widetilde{d}\left(\widetilde{\sigma}_{1}, \widetilde{\sigma}\right)<\varepsilon$ and so $F_{1}=\widetilde{F}_{1} \subset U$.

Now we assume that $F$ is unbounded. Then $\mathrm{bd} F$ is unbounded too and there exist sequences $\left\{x_{r}\right\} \subset \operatorname{bd} F$ and $\left\{y_{r}\right\} \subset \mathbb{R}^{n} \backslash F$ such that $\left\|x_{r}\right\| \rightarrow+\infty$ and $\left\|y_{r}-x_{r}\right\| \rightarrow 0$. Since $\left\{y_{1}, y_{2}, \ldots\right\}$ is closed, $U:=\mathbb{R}^{n} \backslash\left\{y_{1}, y_{2}, \ldots\right\}$ is an open set such that $F \subset U$.

Let $k>0$ such that $\left\|a_{t}\right\|<k$ for all $t \in T$. Let $\sigma_{r} \in \Theta$ be the system obtained from $\sigma$, aggregating $a_{t}^{\prime}\left(y_{r}-x_{r}\right)$ to the RHS coefficient $b_{t}$ for all $t \in T, r=1,2, \ldots$. Denoting by $F_{r}$ the 
solution set of $\sigma_{r}, y_{r} \in F_{r} \backslash U, r=1,2, \ldots$. Since $d\left(\sigma_{r}, \sigma\right) \leq k\left\|y_{r}-x_{r}\right\|$ for all $r, d\left(\sigma_{r}, \sigma\right) \rightarrow 0$ and so $\mathcal{F}$ cannot be usc at $\sigma$.

An immediate consequence of the previous result is that, $\mathcal{F}$ is locally bounded at $\sigma \in \Theta_{c}$ if and only if $F$ is bounded.

Proposition 20 Each of the following conditions guarantees that $\mathcal{F}^{X}$ is usc at $\sigma \in \Theta_{c}^{X}$ :

(i) $\mathcal{F}$ is usc at $\sigma$ and $\mathcal{F}^{X}$ is closed at $\sigma$.

(ii) $F$ is bounded and $F \cap \operatorname{cl} X \subset X$ (e.g., $X$ is closed).

(iii) $F^{X}$ is bounded and $X$ is closed and convex.

(iv) $X$ is compact.

Proof: (i) It is straightforward consequence of Proposition 7(iii).

(ii) $\Longrightarrow$ (i) by Propositions 19 and 10.

(iii) Let $U$ be an open set such that $F^{X} \subset U$. Since $X$ is intersection of closed halfspaces, $X$ is the solution set of some system $\left\{a_{t}^{\prime} x \geq b_{t}, t \in S\right\}$, with $S \cap T=\emptyset$. Consider the system

$$
\widetilde{\sigma}=\left\{a_{t}^{\prime} x \geq b_{t}, t \in W \cup S ; a_{t}^{\prime} x=b_{t}, t \in E\right\},
$$

with associated feasible set mapping $\widetilde{\mathcal{F}}$. Since the solution set of $\widetilde{\sigma}$ is $\widetilde{F}=F^{X}$, and this is bounded, $\widetilde{\mathcal{F}}$ is usc at $\widetilde{\sigma}$ (by Proposition 19). Let $\varepsilon>0$ such that $d\left(\widetilde{\sigma}_{1}, \widetilde{\sigma}\right)<\varepsilon$ implies that its solution set $\widetilde{F}_{1}$ satisfies $\widetilde{F}_{1} \subset U$.

Now, we associate with each $\sigma_{1} \in \Theta$, with solution set $F_{1}$, the system $\widetilde{\sigma}_{1}$ which results of aggregating to $\sigma_{1}$ the inequalities $a_{t}^{\prime} x \geq b_{t}, t \in S$. If $d\left(\sigma_{1}, \sigma\right)<\varepsilon$, we have $d\left(\widetilde{\sigma}_{1}, \widetilde{\sigma}\right)<\varepsilon$ and so $F_{1}^{X}=\widetilde{F}_{1} \subset U$.

(iv) It follows from Proposition 4.

Taking $n$ and $\sigma$ as in Example 6 and $X$ an arbitrary closed subset of $\mathbb{R}, \mathcal{F}$ is usc at $\sigma$ (see Exercise 6.6 in [10]) and $\mathcal{F}^{X}$ is closed at $\sigma$ (by Proposition 10), but $F$ is unbounded. Thus, (i) holds, (ii) fails (so that (ii) is stronger than (i)) and (iii) holds if and only if $X$ is convex depending on $X$. The next result can be seen as a converse of statement (iii) of Proposition 20).

Proposition 21 If $\mathcal{F}^{X}$ is usc at $\sigma \in \Theta_{c}^{X} \cap \widehat{\Theta}, W=\emptyset$ and $X$ is a closed convex such that $\operatorname{dim} 0^{+} X=n$, then $F^{X}$ is either $X$ or a bounded set.

Proof: In order to use matrix notation, we assume that $|E|<\infty$ (the proof is essentially the same for an arbitrary $E)$. Let $\sigma=\{A x=b\}, A(m \times n)$ and $b \in \mathbb{R}^{m}$.

Assume that $F^{X}$ is an unbounded set different of $X$. Consider the open convex set $U:=F^{X}+B\left(0_{n} ; 1\right)$. We must prove that the inclusion $F \subset U$ is not preserved by small perturbations of $\sigma$. 
Since $F \neq \mathbb{R}^{n}$ because $F^{X} \neq X, 0<\operatorname{dim} \operatorname{ker} A<\operatorname{dim} 0^{+} X=n$. On the other hand, $0^{+} F^{X}=(\operatorname{ker} A) \cap 0^{+} X$.

Take $\bar{x} \in F^{X}, y \in 0^{+} F^{X}$, and $z \in\left(0^{+} X\right) \backslash$ ker $A$ such that $y \neq 0_{n} \neq z$. Let $M(n \times n)$ be such that $M y=z$. The matrix $r I_{n}+M$ is non-singular for $r$ big enough, say $r \geq r_{0}$.

Given $r \in \mathbb{N}$, we define $\sigma_{r}:=\left\{A_{r} x=b_{r}\right\}$, with solution set $F_{r}$, such that

$$
A_{r}=A\left[I_{n}-M\left(r I_{n}+M\right)^{-1}\right]
$$

and $b_{r}=A_{r} \bar{x}$. We have $\bar{x} \in F_{r}^{X}$,

$$
A_{r}-A=-A M\left(r I_{n}+M\right)^{-1}
$$

and $b_{r}-b=\left(A_{r}-A\right) \bar{x}$. Moreover, $y+\frac{z}{r} \in \operatorname{ker} A_{r}$ and $y+\frac{z}{r} \in 0^{+} X$, so that $y+\frac{z}{r} \in 0^{+} F_{r}^{X}$. Nevertheless, since

$$
A\left(y+\frac{z}{r}\right)=\frac{1}{r} A z \neq 0_{m}
$$

$y+\frac{z}{r} \notin(\operatorname{ker} A) \cap 0^{+} X=0^{+} F^{X}=0^{+} U$. Since $0^{+} F_{r}^{X} \nsubseteq 0^{+} U, F_{r}^{X} \nsubseteq U$.

Observing that $F_{r}^{X} \nsubseteq U$ for $r \geq r_{0}$ whereas $d\left(\sigma_{r}, \sigma\right) \rightarrow 0$, we conclude that $\mathcal{F}^{X}$ cannot be usc at $\sigma$.

\section{Conclusions}

This paper provides sufficient conditions and necessary conditions for the closedness, the lower and the upper semicontinuity of the feasible set mapping $\mathcal{F}^{X}$ of a system $\sigma$ with inequality constraints, equations and exact constraint set. The paper characterizes the closedness of $\mathcal{F}^{X}$ for the class of systems with bounded LHS function and proves the closedness of $\mathcal{F}^{X}$ when $X$ is closed. The lower (upper) semicontinuity at the nominal system has been characterized in some cases, e.g., when the coefficient function (the LHS function, respectively) is bounded and $X=\mathbb{R}^{n}$.

Concerning the viability of checking in practice the conditions in this paper, let us observe that there exists some SS point of $\sigma$ if and only if the linear system

$$
\left\{a_{t}^{\prime} x-x_{n+1} \geq b_{t}, t \in W ; a_{t}^{\prime} x \geq b_{t}, t \in E ; x_{n+1}>0\right\}
$$

is consistent. If $X$ is the solution set of some convex system

$$
\left\{f_{s}(x)<0, s \in S ; f_{s}(x) \leq 0, s \in Z\right\}
$$

the existence of SS point of $\sigma$ in $X$ is equivalent to the consistency of the aggregation of (2) and (3). Consistency tests for such kind of systems are discussed in [7]). Other conditions are formulated in terms of the containment of two sets. For instance, $F \cap c l X \subset X$ (the sufficient condition in Proposition 10) holds if and only if 


$$
\begin{gathered}
\left\{x \in \mathbb{R}^{n} \mid a_{t}^{\prime} x \geq b_{t}, s \in W ; a_{t}^{\prime} x=b_{t}, s \in E ; f_{s}(x) \leq 0, s \in S \cup Z\right\} \\
\subset\left\{x \in \mathbb{R}^{n} \mid f_{s}(x)<0, s \in S ; f_{s}(x) \leq 0, s \in Z\right\} .
\end{gathered}
$$

The containment of the solution sets of pairs of systems as those in (4) has been also characterized in [7]. Most conditions become very simple when $\sigma$ is an ordinary linear systems. As an illustration, consider the LP models $P_{1}, P_{2}, P_{3}$ introduced in Section 1, whose constraint systems, denoted by $\sigma_{1}, \sigma_{2}, \sigma_{3}$, we assume to be consistent.

The feasible set mapping $\mathcal{F}^{X}$ is closed for all $i$ because $X$ is closed (Corollary 12). Moreover, $\mathcal{F}^{X}$ is lsc at:

- $\sigma_{1}$ if and only if $\{A x>b, B x=d\}$ is consistent and $B$ is full-row rank (Proposition 14).

- $\sigma_{2}$ if and only if $\mathbb{R}_{++}^{n}$ contains some solution of $\{A x=b\}$ and $A$ is full-row rank (by Propositions 13 and 17, because rint $F=F$ and rint $\left.\mathbb{R}_{+}^{n}=\mathbb{R}_{++}^{n}\right)$.

- $\sigma_{3}$ if and only if any solution of $\sigma_{3}$ in $\{0,1\}^{n}$ satisfies $A x>b$ (Corollary 18).

Finally, $\mathcal{F}^{X}$ is usc at:

- $\sigma_{1}$ if and only if $F$ is either $\mathbb{R}^{n}$ (i.e., $A$ and $B$ are null matrices, $b \in \mathbb{R}_{-}^{n}$ and $d=0_{n}$ ) or a bounded set (Proposition 19).

- $\sigma_{2}$ if and only if $F^{X}$ is either $\mathbb{R}_{+}^{n}$ or a bounded set (Propositions 20 and 21).

- $\sigma_{3}$, due to the compactness of $X$ (Proposition 4).

The results in Sections 4-6 could be useful in order to study the stability properties of the optimal set and the optimal value mappings for linear optimization problems with equations and inequalities subject to perturbations and a fixed constraint set.

\section{References}

[1] Amaya, J., Goberna, M.A.: On the stability of linear systems with an exact constraint set. Math. Methods Oper. Res. 63, 107-121 (2006).

[2] Aubin, J.-P., Frankowska, H.: Set-Vaued Analysis. Birkhäuser, Boston (1990).

[3] Bank, B., Guddat, J., Klatte, D., Kummer, B., Tammer, K.: Non-Linear Parametric Optimization. Birkhäuser, Basel (1983).

[4] Berge, C.: Topological Spaces. Dover, Mineola, NY (1997).

[5] Cánovas, M.J., López, M.A., Parra, J.: Upper semicontinuity of the feasible set mapping for linear inequality systems. Set-Valued Analysis 10, 361-378 (2002). 
[6] Cánovas, M.J., Gómez-Senent, F.J., Parra, J.: Stability of systems of linear equations and inequalities: distance to ill-posedness and metric regularity. Optimization, to appear.

[7] Goberna, M.A., Jeyakumar, V., Dinh, N.: Dual characterizations of set containments with strict convex inequalities. J. Global Optim. 34, 33-54 ( 2006).

[8] Goberna, M.A., Larriqueta, M., Vera de Serio, V.N.: On the stability of the boundary of the feasible set in linear optimization. Set-Valued Analysis 11, 203-223 (2003).

[9] Goberna, M.A., Larriqueta, M., Vera de Serio, V.N.: Stability of the intersection of solution sets of semi-infinite systems. J. Comp. Appl. Math., to appear.

[10] Goberna, M.A., López, M.A.: Linear Semi-Infinite Optimization. J. Wiley, Chichester (1998).

[11] Goberna, M.A., López, M.A., Todorov, M.I.: Stability theory for linear inequality systems. SIAM J. Matrix Anal. Appl. 17, 730-743 (1996).

[12] Goberna, M.A., López, M.A., and Todorov, M.I.: A generic result in linear semi-infinite optimization. Appl. Math. Optim. 48, 181-193 (2003).

[13] Goberna, M.A., López, M.A., Todorov, M.I.: On the stability of closed-convex-valued mappings and the associated boundaries. J. Math. Anal. Appl. 306, 502-515 (2005).

[14] Hogan, W.W: Point-to-set maps in mathematical programming. SIAM Rev. 15, 591-603 (1973).

[15] Jongen, H.T., Rückmann, J.-J.: On stability and deformation in semi-infinite optimization. In: Reemtsen, R., Rückmann, J.-J. (eds.) Semi-Infinite Programming, pp. 29-67. Kluwer, Dordrecht (1998).

[16] Jongen H.T., Twilt, F., Weber. G.-H.: Semi-infinite optimization: structure and stability of the feasible set. J. Optim. Theory Appl. 72, 529-552 (1992).

[17] Klatte, D., Henrion, R.: Regularity and stability in nonlinear semi-infinite optimization. In: Reemtsen, R., Rückmann, J.-J. (eds.) Semi-Infinite Programming, pp. 69-102. Kluwer, Dordrecht (1998).

[18] Klatte, D., Li, W.: Asymptotic constraint qualifications and global error bounds for convex inequalities. Math. Programming 84, 137-160 (1999).

[19] Rockafellar, T., Wets, R.: Variational Analysis. Springer, Berlin (1998). 